\title{
L-band 4×4 Full-redundant Matrix Based on Microwave Multilayer Technique
}

\author{
Xiaoming $\mathrm{Li}^{a}$, Qinghui Song, and Jiangkun $\mathrm{Du}$ \\ The 54th Research Institute of CETC, Shijiazhuang 050081, Hebei, P.R. China
}

\begin{abstract}
A planar RF $4 \times 4$ microwave switch matrix has been proposed to reduce the size of traditional RF matrix at L-band, where multilayer microwave technique based on PCB is employed to integrate embedded power divider and RF interconnections. Buried components are simulated and optimized using 3D EM software to obtain good circuit performance. A prototype of the matrix is designed and fabricated on Arlon "CLTE-XT" substrate with "25N" prepreg. For all ports of the matrix and among the working band from $950 \mathrm{MHz}$ to $2150 \mathrm{MHz}$, both input and output VSWR are less than 1.7, transmission gains are between 6.8 and 8 , while ON-OFF isolations are better than $50 \mathrm{~dB}$. The dimension of the matrix is not greater than $70 \mathrm{~mm} \times 120 \mathrm{~mm} \times 20 \mathrm{~mm}$, which is reduced by 2 order of magnitude compare with traditional design.
\end{abstract}

\section{Introduction}

Microwave switch matrix (MSM) is a frequently used device in modern electric systems, especially in multibeam systems [1]. Performance of MSM playing an important role in system behaviour, it got continuously attention in both academic and industry fields. François Assal in COMSAT Lab. and Xavier Rozec in Thomson Corp. proposed a lightweight, low-power-consuming $8 \times 8$ MSM [2] in 1979, it has implemented cross-bar configuration to provide full interconnect flexibility, and use push-pull connectors to ease the assembly procedure. Although the matrix of François and Xavier seems bulky in nowadays, the structure is still popular due to its advantage on modulization and expanding ability. R. K. Gupta in COMSAT proposed another type of $4 \times 4$ quasiplanar MSM in 1988 [3], it is composed of input plane, output plane, metal structure between them and connections. Gupta's structure provides much smaller size compare with the former one, but the arrangement of its input and output port is not convenient for combination. Recent research on matrix are concentrates on new integration platform like SIP or SOC, but none of them are fully-redundant $[4,5]$.

With rapidly development of MIC and MMIC, more and more attentions are transferred to planar circuit in RF design, which can reduce system size and cost tremendously. It is obviously that the bottleneck of planar circuit miniaturization is component mounting area, which is equal to top and bottom layer area in traditional design. Microwave multilayer technique [6, 7] is developed to deal with the problem, it is focused on use of inner layer of PCB, LTCC and other planar circuit platforms. As many microwave components like filter, power divider, and coupler are realized via transmission line, it is convenient to integrate in planar circuit platform directly. In this paper, design method of microwave multilayer technique based on PCB is discussed, a $4 \times 4$ Full-redundant MSM work in L-band is designed using the method, which exhibit good RF performance and occupy very small circuit area. In addition, the proposed MSM have the ability to rearrange $\mathrm{RF}$ port conveniently.

\section{System design of MSM}

\subsection{Topology}

In order to meet the requirement of full-redundant, topology in figure 1 is frequently used for $\mathrm{N} \times \mathrm{M}$ MSM. Typical MSM is composed of $\mathrm{N}$ M-way divider module, $\mathrm{M} N$-way switch module and $\mathrm{N} \times \mathrm{M}$ connection $\mathrm{RF}$ cables between them. The input of M-way power divider is connected to each input port of the matrix, while $\mathrm{M}$ outputs of divider are connected to corresponding switch module. The output of $\mathrm{N}$-way switch module is connected to each output port of the matrix. In practice, switch module usually use two or more stage of MMIC switch to improve isolation between different channels.

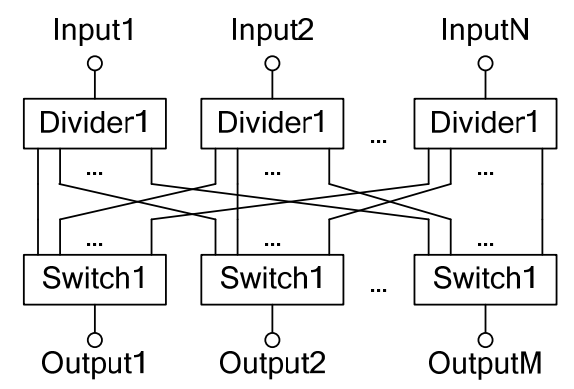

Fig. 1. Topology of full-redundant MSM

\footnotetext{
${ }^{a}$ Corresponding author: 624910659@qq.com
} 


\subsection{Structure}

PCB platform is selected for multilayer layout to reduce costs. Symmetry for both input and output is chosen so unitary divider and switch module are employed. Four buried Wilkinson 4-way equal divider is used to provide isolation between outputs connected to same input. Two stage MMIC switch is cascaded to construct 4-way switch module. Sketch map of the proposed structure is illustrated in figure 2. four Wilkinson 4-way dividers are stacked in the inside of the PCB board, top and bottom layer of the board are used to mounting Dc-Dc module, control unit, switch module, and amplifier modules. Buried divider occupied no surface area and can provide good VSWR and insertion loss compare with chip divider. These characters make superiority of the proposed MSM both on size and RF performance.

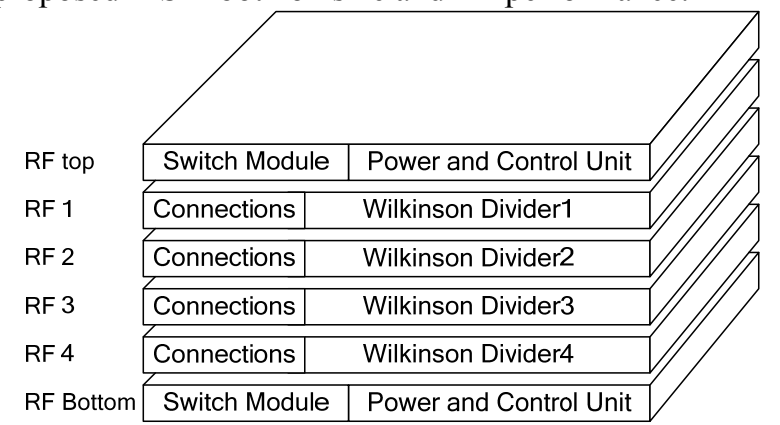

Fig. 2. Structure sketch map of proposed MSM

In figure 2, four buried RF layers and two component layers are stacked together, so six RF layers in all are designated.

\section{Material system and laminates design}

\subsection{Material system}

Based on comprehension consideration of cost, size, performance, and the ability of buried resistor integration, the laminate material "CLTE-XT" and prepreg material " $25 \mathrm{~N}$ " produced by Arlon Corp. are selected. "CLTEXT" has a relative dielectric constant of 2.94 , loss tangent of $0.0012, \mathrm{x}$ and $\mathrm{y}$ axis CTE of 8, $\mathrm{z}$ axis CTE of 20. " $25 \mathrm{~N}$ " has a relative dielectric constant of 3.38 , loss tangent of $0.0025, \mathrm{x}$ and $\mathrm{y}$ axis CTE of $15, \mathrm{z}$ axis CTE of 50. Two laminates and one prepreg composite a single buried RF layer illustrated in figure2.

\subsection{Laminates design}

As well known, for each buried RF layer or component layer, thicker laminates and prepreg can provide more impedance precise under fabrication, but it also introduced larger circuit size. Considered that impedance precise can influence the RF performance, miniaturization and RF performance forms a trade-off on circuit design. Another restriction on laminate design is the total thickness of PCB board, fabrication of $6 \mathrm{~mm}$ thick or above PCB usually causes an unacceptable price. Under these considerations, the thickness of the laminate is chosen to be $0.254 \mathrm{~mm}$, while the thickness of prepreg is set to be $0.1 \mathrm{~mm}$. PCB stack scheme of the proposed MSM is illustrated in figure 3. Totally 10 laminates and 9 prepregs are used to form a 20 layer PCB structure. Thickness of copper foil cladding is $1 / 2 \mathrm{oz}$ for inner layers and $1 \mathrm{oz}$ for component layers. 9 types of via are used in the design which can be degenerated to 5 types in process flow because its symmetry. Totally thickness of the board is below $4 \mathrm{~mm}$, which is economic applicable in fabricate.

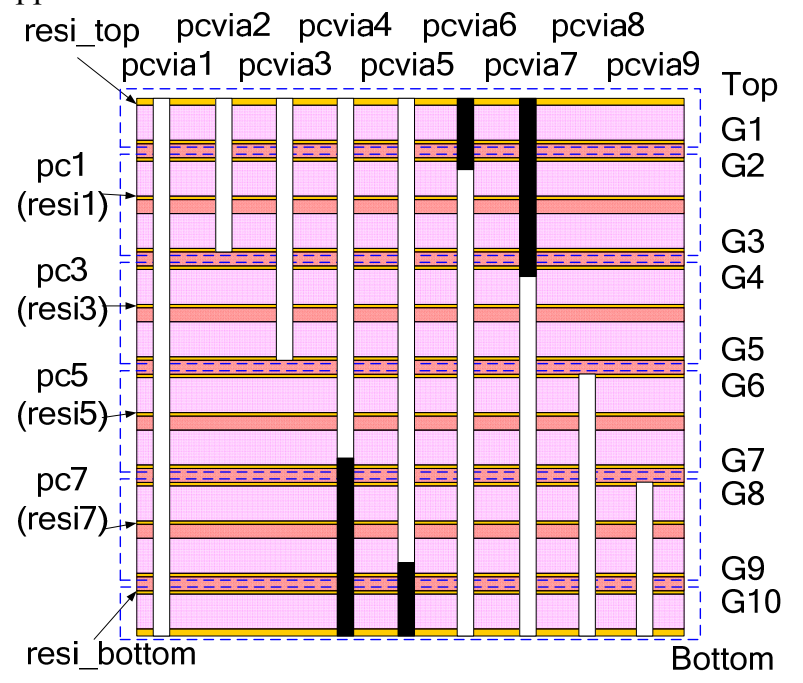

Fig. 3. PCB stack scheme of proposed MSM

\section{Design of buried transmission line and passive components}

\subsection{Buried transmission line}

Transmission line (TL) is the most basic element of RF circuits, different components are connected by TL and some RF components are composed of TLs it self. Typical buried TL is somewhat like strip line (SL) or eccentric strip line (ESL) but not the same. The key distinction between buried TL and SL or ESL is that the dielectric around signal line is inhomogeneous, which is illustrated in figure 4 .

\begin{tabular}{|c|c|c|}
\hline $0.254 \mathrm{~mm}$ & $\stackrel{w_{0}}{\longrightarrow}$ & CLTE-XT \\
\hline $0.1 \mathrm{~mm}$ & & $25 N$ \\
\hline $0.254 \mathrm{~mm}$ & & CLTE-XT \\
\hline
\end{tabular}

Fig. 4. Cross-section of buried transmission line

The boundary condition of inhomogeneous dielectric cross-section in figure 4 dose not allow pure TEM mode. Consequently, dispersion is expected in the buried TL. Fortunately, characters between laminate and prepreg in this paper are not large enough and the working frequency is relatively low, so the dispersion is not as bad as to take into account. Another problem is that design parameters of buried TL, like characteristic impedance and effective dielectric constant can not be 
calculated via expressions in existence. An effective way to get buried TL design parameters is to solve the Maxwell equations on the cross-section by numerical method, several commercial EM softwares can be used to do this. This paper use ANSYS EM to compute characteristic impedance and effective dielectric constant vary with strip width $w_{0}$, simulation results are shown in figure 5(a) and (b) respectively. The intersection point of $50 \mathrm{ohm}$ grid and impedance curve is marketed on figure 5 (a), $x$ coordinate of the point is the width of $50 \mathrm{ohm} \mathrm{TL.}$ Using the same method, effective dielectric constant of $50 \mathrm{ohm}$ buried TL can be read from figure 5(b), which is about 3.03 .

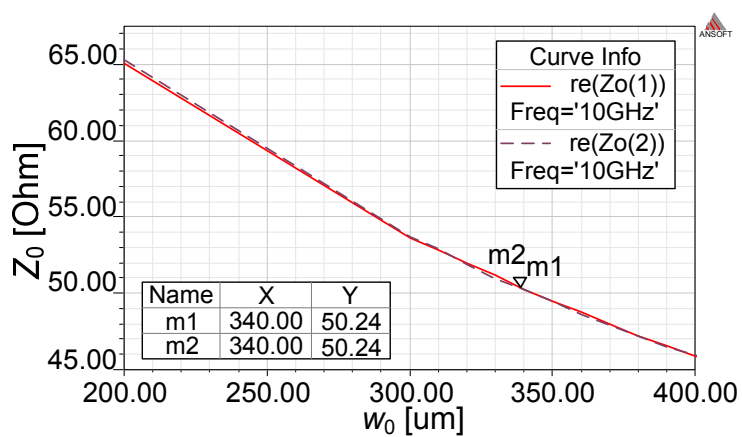

(a) Characteristic impedance with $w_{0}$

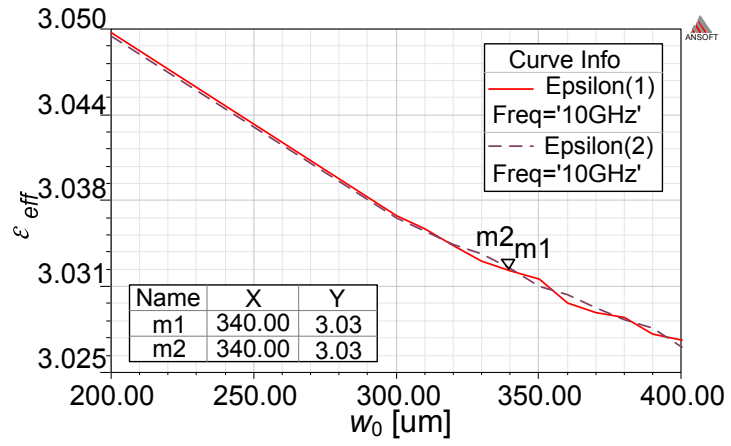

(b) Effective dielectric constant with $w_{0}$

Fig. 5. Simulation results of buried TL using ANSYS EM

\subsection{Buried Wilkinson divider}

Buried 4-way Wilkinson divider is composed of three 2-way divider, witch model is illustrated in figure 6 (a). The simulation of 2-way divider is also made by ANSYS EM, simulation results are shown in figure 6(b)-(d).

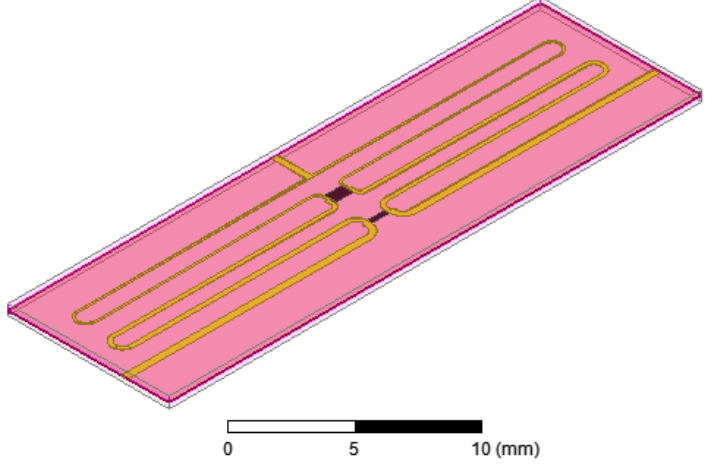

(a) Simulation model of 2-way divider

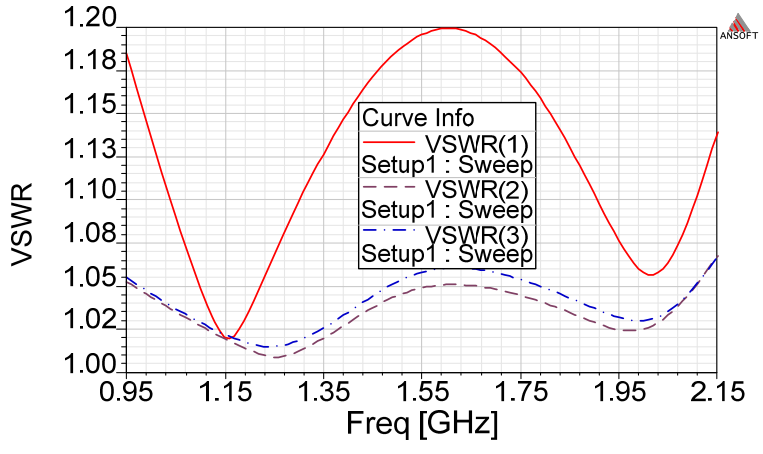

(b) VSWR of 2-way divider

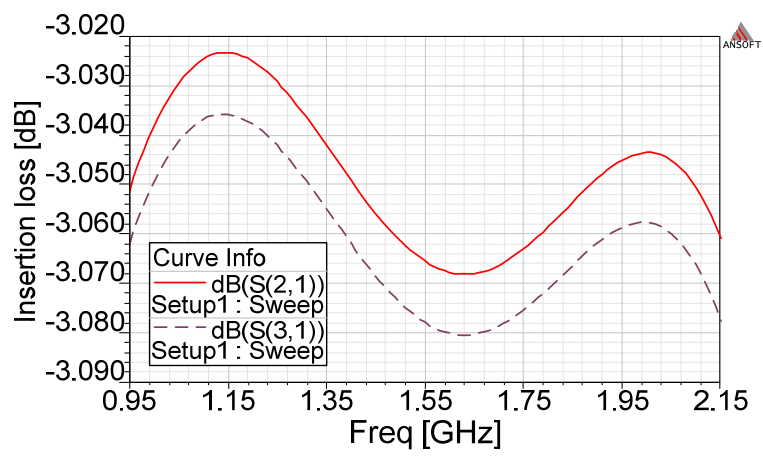

(c) Insertion loss of 2-way divider

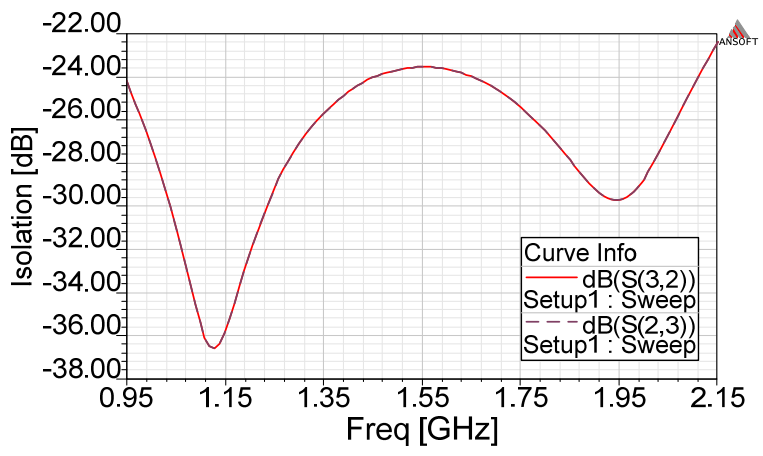

(d) Isolation of outputs of 2-way divider

Fig. 6. Model and simulation results of 2-way Wilkinson divider

\section{Fabrication and measurement}

A prototype of proposed MSM is fabricated based on former designs. The pictures of its mother board and MSM itself are given in figure 7(a) and (b). The matrix is measured by Agilent vector network analyser N5230A, which is calibrated by $85052 \mathrm{D}$ calibration kit. Measurement results are in figure 7(c)-(f). It can be seen that the VSWR of both input and output is less than 1.7; the transmission gain of the on-state is between 6.8 and 8 . The off-state insertion loss is greater than $45 \mathrm{~dB}$, which leads a on-off isolation (on-state transmission gain plus off-state insertion loss) more than $50 \mathrm{~dB}$. 


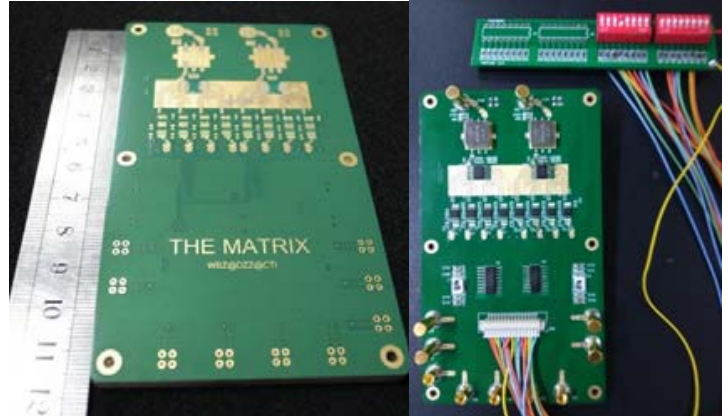

$\begin{array}{ll}\text { (a) Mother board of MSM } & \text { (b) Control environment }\end{array}$

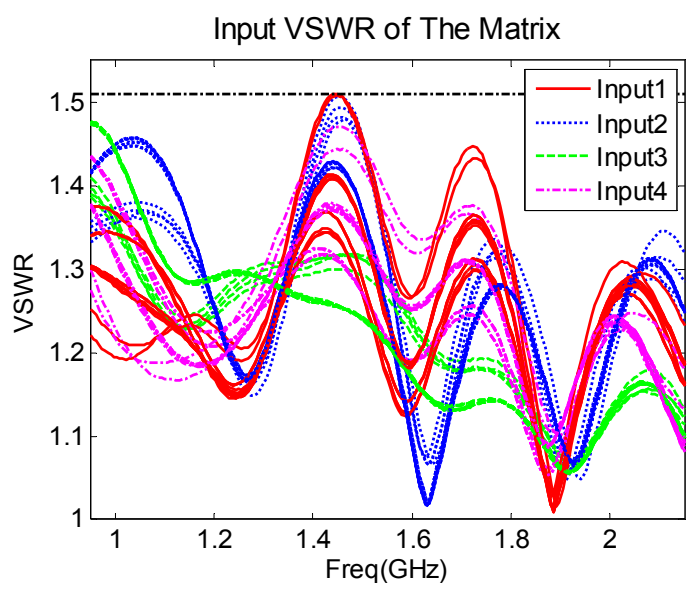

(c) VSWR of the input ports

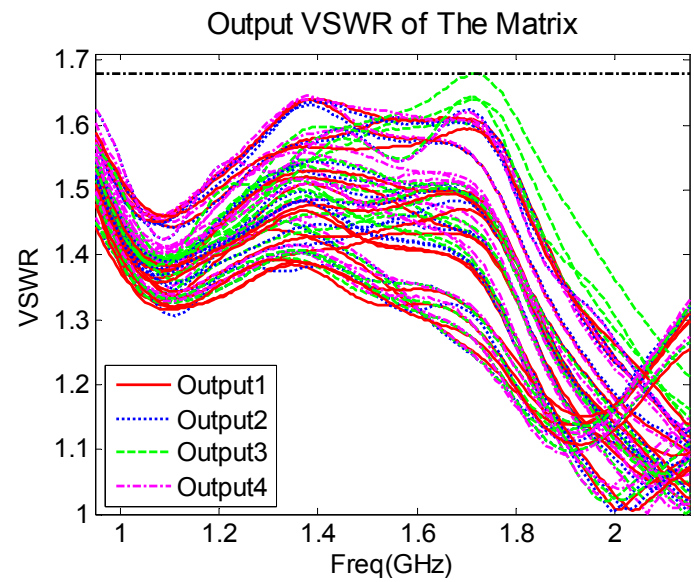

(d) VSWR of the input ports

$\mathrm{S}_{21}$ of The Matrix (On)

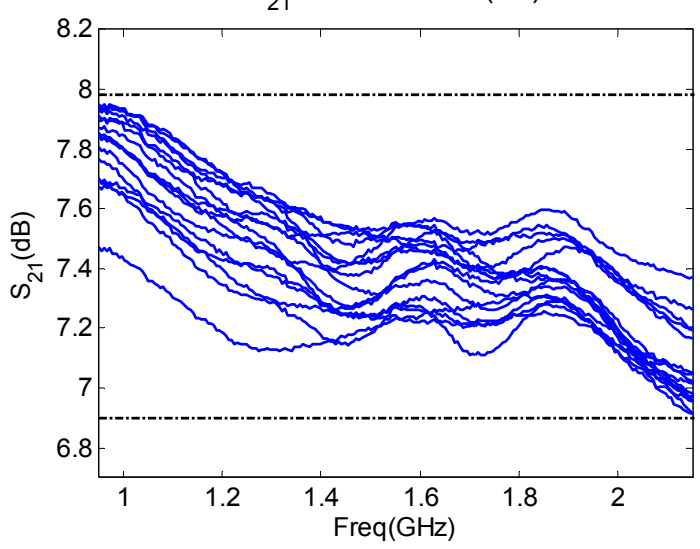

(e) Insertion loss of the on-state

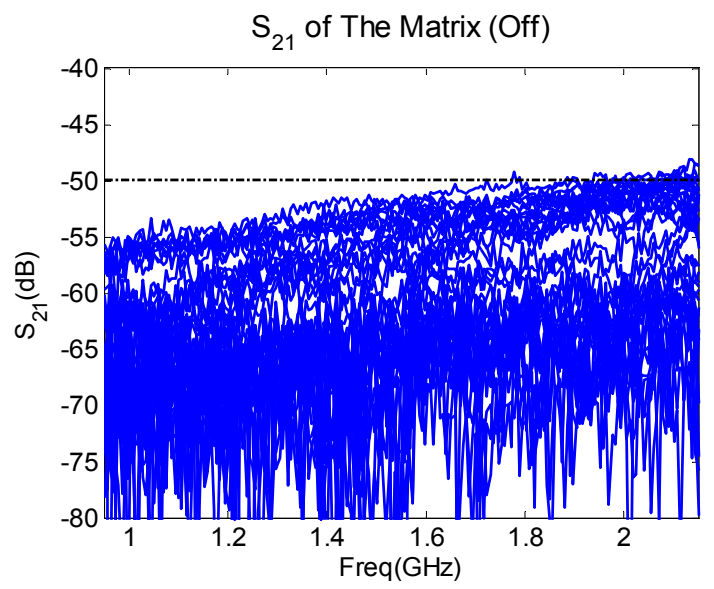

(f) Off-state insertion loss of the MSM

Fig. 7. Picture and measurements of the proposed MSM

\section{Conclusion}

In this paper, a novel L band planar $2 \times 2 \mathrm{MSM}$ based on microwave multilayer technique is proposed. Prototype of the MSM is designed and realized via PCB technique. The measurements of the MSM shown good performance on VSWR, transmission gain and on-off isolations, while a tremendous size reduce is achieved compare with traditional way. Benefits from mature PCB technique, the proposed MSM also have the character of low cost. It is obviously that the proposed MSM have potential application prospects in various communication systems, especially in space borne environment.

\section{References}

1. L. Qinghua, L. Pei, L. Xiao, etc. A prototype of feed subsystem for a mutilple-beam array-fed reflector antenna. ISAP (2015), 1-3.

2. F. Assal, X. Ro. Fast, Fully-Redundant, 4 GHz, 8x8 Microwave Switch Matrix for Communications Satellites. 9th EMC (1979), 218-222.

3. Gupta R. K., Gerson H. I., Ross P. B., etc. Design and packaging approach for MMIC insertion in a broadband $4 \times 4$ microwave switch matrix. GaAs IC Symposium (1988), 261-264.

4. S. Kaleem, S. Rentsch, S. Humbla, etc. Highly integrated reconfigurable microwave switch matrix module for geostationary satellites. 44th EMC (2014), 992-995.

5. A. Ebert, S. Kaleem, J. Müller. An industry-level implementation of a compact microwave diode switch matrix for flexible input multiplexing if a geo-stationary satellite payload. COMCAS (2015), 1-4.

6. Metzen P L. Globalstar Satellite Phased Array Antennas. ICPAT (2000), 207-210.

7. Gustafasson A, Malmqvist R, Pettersson Lz. A Very Thin and Compact Smart Skin X-Band Digital Beamforming Antenna. European Radar Conference (2004), 313-316. 